\title{
Computational hemodynamics of portal vein hypertension in hepatic cirrhosis patients
}

\author{
X. Li ${ }^{\mathrm{a}}$, X.K. Wang ${ }^{\mathrm{a}}$, B. Chen ${ }^{\mathrm{a}, *}$, Y.S. Pu ${ }^{\mathrm{b}}$, Z.F. Li ${ }^{\mathrm{b}}, \mathrm{P} . \mathrm{Nie}^{\mathrm{c}}$ and K. $\mathrm{Su}^{\mathrm{c}}$ \\ ${ }^{a}$ State Key Laboratory of Multiphase Flow in Power Engineering, Xi'an Jiaotong University, Xi'an \\ City, Shaanxi Province, P.R. China \\ ${ }^{b}$ Second Affiliated Hospital, Xi'an Jiaotong University, Xi'an City, Shaanxi Province, P.R. China \\ ${ }^{c}$ Department of Radiology, Huaihe Hospital Affiliated to Henan University, Kaifeng City, Henan \\ Province, P.R. China
}

\begin{abstract}
Portal vein hypertension generally occurs in liver diseases like hepatic cirrhosis. It causes hemodynamic changes that are closely related to liver disease. At advanced stages of hepatic cirrhosis, portal vein hypertension leads to the atrophy of the right lobe of the liver and the hypertrophy of the left lobe through a process that has not yet been fully explained. Based on the hemodynamic changes that are known to occur, we hypothesize that liver volume is related to the distribution of blood flowing from the splenic vein (SV) that carries hepatotrophic factors from the spleen and pancreas. We studied blood flow in the portal vein system to validate this hypothesis through in vitro experimentation and a computational fluid dynamics (CFD) analysis involving both simplified and patient-specific models based on four healthy subjects and two patients with liver cirrhosis. The results confirmed the hypothesis that right-lobe atrophy is significantly influenced by the distribution of blood from the SV. Moreover, the patients with liver cirrhosis had a significantly larger mass fraction of spleen-derived blood in the left portal vein branch (LPV) than healthy subjects, a result consistent with right-lobe atrophy and left-lobe hypertrophy.
\end{abstract}

Keywords: Portal vein hypertension, hemodynamics, hepatotrophic factor, splenic vein

\section{Introduction}

The portal vein is the liver's main blood vessel, supplying $70 \%-80 \%$ of blood that passes through the liver [1] from the digestive tract, spleen, pancreas and gallbladder. The portal system transports abundant nutrients into the liver, not only to provide energy for the liver's metabolism but also to synthesize new substances for use by other tissues. The portal vein system is composed of the superior mesenteric vein (SMV) and SV joining to form the portal vein (PV), which then divides into the LPV and the right portal vein branch (RPV) entering the left and right lobes of the liver, respectively [2]. The portal system delivers nutrients from the intestine through the SMV and hormones from the pancreas and spleen through the SV into the liver, where nutrient metabolism and detoxification take place [3].

\footnotetext{
* Address for correspondence: Bin Chen, State Key Laboratory of Multiphase Flow in Power Engineering, Xi'an Jiaotong University, Xi'an City, Shaanxi Province, 710049, P.R. China. Tel.: +86-29-82667326; Fax: +86-29-82669033; E-mail: chenbin@mail.xjtu.edu.cn.
} 
Liver cirrhosis creates resistance to blood flow through the portal system, causing persistent increased portal vein pressure known as portal hypertension [4]. When pressure in the PV increases, ramus communicans between the PV and the vena systemic may form. Then a large amount of blood intended to flow through the PV may instead flow into systemic circulation, bypassing the liver and resulting in abdominal wall and esophageal vein dilation, splenomegaly and hypersplenism, hepatic decompensation, and ascites. Gastroesophageal varices present the most serious consequence of this process. If these varices break, they can cause acute hemorrhage in the upper digestive tract that is often fatal $[5,6]$.

At advanced stages of hepatic cirrhosis, portal hypertension leads to the atrophy of the right lobe of the liver and the hypertrophy of the left lobe [7], a phenomenon which has not yet been definitively explained. Liver volume is directly related to liver function and the patient's prognosis [8]. Thus, patients with serious liver atrophy usually have poor liver function and liver reserve function, and therefore they are prone to hepatic decompensation and liver failure. The right lobe's volume represents two-thirds of the total liver volume; thus, it is critical to prevent right hepatic lobe atrophy in order to improve liver function in patients with cirrhosis and portal hypertension.

As described above, the PV supplies the liver with enterogenous blood flow from the SMV and spleen blood flow from the SV. Enterogenous blood flow contains nutrients absorbed from the intestine which are delivered to the liver for further processing, biological transformation and utilization. Due to the joining of the venae pancreaticae, blood traveling from the spleen contains hormones, like insulin and glucagon that are known to promote hepatocyte proliferation $[9,10]$. Consequently, it seems reasonable to propose that liver volume is related to the distribution of blood from the SV, which carries hepatotrophic substances from the spleen and pancreas. Under normal circumstances, the blood flowing from the SV through the PV is uniformly distributed between the left and right lobes. However, when liver cirrhosis causes changes in the PV, the distribution of blood from the SV changes. This can result in the routing of more hepatotrophic substances to the left lobe and less to the right lobe, which may lead to the atrophy of the right lobe and the hypertrophy of the left lobe. However, proving this hypothesis by in vivo experimentation is difficult.

In recent years, CFD has shown great potential in computational biology and biomechanics due to the limitations of traditional measurement methods. In the study of portal system hemodynamics, Botar, et al. [11] implemented the Reynolds stress model to describe hemodynamics in the PV system. Petkova, et al. [12] examined blood flow in idealized models of the PV with and without thrombosis to evaluate its impact on the flow behavior. George [13] employed an idealized PV model to simulate how the blood coming from the SV and SMV distributes between the left and right lobes of the liver.

To our knowledge, numerical simulation of the distribution of PV blood flow and the validation of the hepatotrophic factor hypothesis have not been undertaken to date. In this study, we constructed an idealized PV model and specific PV models based on the CT images acquired from four normal subjects and two subjects with liver cirrhosis to validate this hypothesis through computational hemodynamics. After validating the algorithm by in vitro experiment, we calculated the mass fraction of blood from the SV in each branch of the PV to test the hypothesis of hepatotrophic factor distribution.

\section{Methods}

\subsection{Idealized portal vein model}




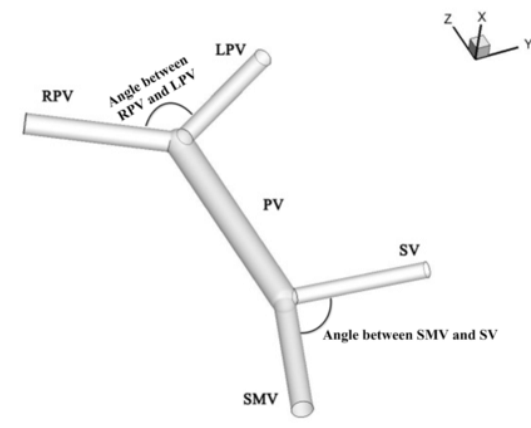

Fig. 1. Geometry of idealized model.

Table 1

Geometric parameters of the idealized model [13]

\begin{tabular}{lll}
\hline Vein & Diameter $/ \mathrm{m}$ & Length $/ \mathrm{m}$ \\
\hline PV & $0.0090-0.0150$ & 0.09 \\
RPV & $0.0074-0.0083$ & 0.06 \\
LPV & $0.0073-0.0075$ & 0.06 \\
SMV & $0.0086-0.0109$ & 0.06 \\
SV & $0.0064-0.0085$ & 0.06 \\
\hline
\end{tabular}

In the preliminary investigation, we developed an idealized but physically representative model of the PV system. As shown in Figure 1, the model consists of the SMV and SV joining to form the PV, which then divides into the LPV and RPV. The vessel parameters, which are listed in Table 1, were obtained from literature. The angle between the SMV and SV is from $90^{\circ}$ to $175^{\circ}$, while the angle between the LPV and RPV is from $80^{\circ}$ to $120^{\circ}$. The cell number in the idealized models is from 525895 to 680691 . We constructed and meshed the idealized models using GAMBIT 2.4.0 with tetrahedral grids before importing them to Fluent 6.3 to calculate the blood flow field.

The idealized models were divided into a normal group and a cirrhosis group. The diameters of the PV, RPV, LPV, SMV, SV in the normal group were $10.8 \mathrm{~mm}, 8.3 \mathrm{~mm}, 7.3 \mathrm{~mm}, 8.6 \mathrm{~mm}$, and $6.4 \mathrm{~mm}$, while in the cirrhosis group they were $14.0 \mathrm{~mm}, 7.4 \mathrm{~mm}, 7.5 \mathrm{~mm}, 10.9 \mathrm{~mm}, 8.5 \mathrm{~mm}$, respectively. We compared the normal group diameters with the experimental results to validate our numerical method, and we compared the cirrhosis group diameters with the normal group to validate the hypothesis about hepatotrophic substances.

\subsection{Patient-specific models}

In order to simulate the hemodynamics of real PV systems, we constructed patient-specific models from two patients with liver cirrhosis and a set of four subjects with normal livers. Data from all the subjects were collected through computed tomography (CT, Toshiba Aquilion ONE 320, Japan) in Huaihe Hospital, affiliated with Henan University. Scans occurred with the subjects in a supine position, and the scanning area extended from the SMV to the bifurcation of the first porta hepatic. For each subject, the process produced 320 layers of scanning images with a thickness of $0.5 \mathrm{~mm}$ in the scanning time of 0.35 seconds. The CT images, which adhered to the Digital Imaging and Communications in Medicine (DICOM) standard, were used to reconstruct a three-dimensional model of the portal vein system. The four subjects in the control group had no history of liver disease, and the livers were all functioning normally. The two patients with liver cirrhosis differed in that Patient 1's 


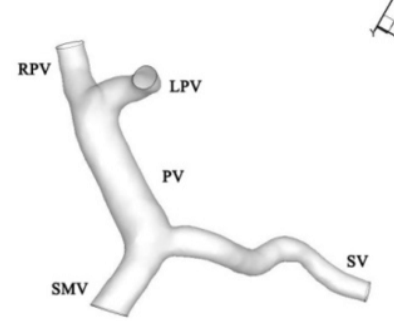

(a) normal subject

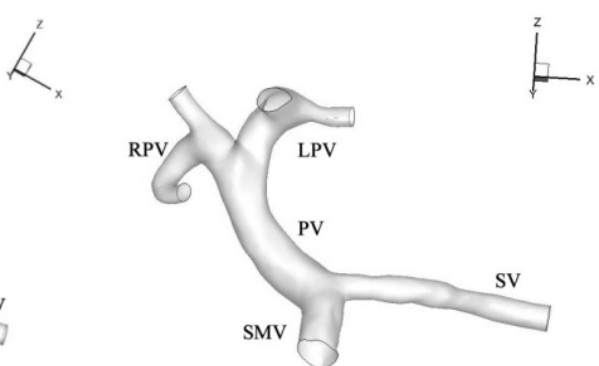

(b) patient 1

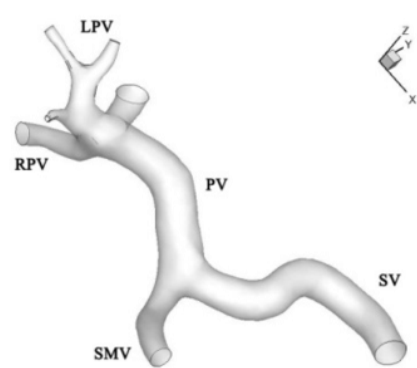

(c) patient 2

Fig. 2. Patient-specific models.

morphological structure showed no obvious changes in the PV system, whereas Patient 2's right hepatic lobe had atrophied and the SV had obviously expanded.

Mimics software reconstructed the geometry of the PV system models for CFD simulation, as shown in Figure 2. To facilitate the hemodynamic calculations, some small parts of the threedimensional reconstruction of the PV system were pruned, retaining only the PV and its main branch vessels including the LPV, RPV, SMV and SV. The finite element analysis (FEA) module of Mimics meshed the geometric models, which were then imported into FLUENT 6.3 to calculate the blood flow field. The cell numbers of the reconstructed models ranged from 50000 to 70000 .

\subsection{Hemodynamics modelling}

Blood in the PV system demonstrates laminar flow with a Reynolds number ranging from 638 to 730. Because the PV is far from the heart, the influence of the cardiac cycle can be disregarded. For simplicity, vessel walls were assumed to be rigid, and blood was considered to behave as a Newtonian fluid.

The mass and momentum conservation equations for three-dimensional incompressible laminar flow are written as follows:

$$
\begin{gathered}
\nabla \cdot \mathbf{u}=0 \\
\nabla \cdot(\rho \mathbf{u u})=\operatorname{div}(\rho v \mathbf{g r a d u})-\nabla p+\rho \boldsymbol{g}
\end{gathered}
$$

where $\rho, p, \mathrm{u}$ are the fluid density, pressure and velocity, respectively. The boundary conditions used in all models were a constant flat profile inlet velocity for the SMV and SV. The average axial velocities of blood in the SMV and SV for the control group were respectively $0.210 \mathrm{~m} / \mathrm{s}$ and 0.179 $\mathrm{m} / \mathrm{s}$, while the average velocities of blood in the SMV and SV for the cirrhosis patients were $0.175 \mathrm{~m} / \mathrm{s}$ and $0.182 \mathrm{~m} / \mathrm{s}$, respectively. The outflow was pre-defined according to the literature, with flow rate weighting 0.6 for the RPV and 0.4 for the LPV [13]. A non-slip boundary condition was implemented at the vessel wall.

The distribution of spleen-derived blood can be obtained from the following convection diffusion equation:

$$
\frac{\partial}{\partial t}\left(\rho Y_{i}\right)+\nabla \cdot\left(\rho \vec{v} Y_{i}\right)=-\nabla \cdot \vec{J}_{i}+R_{i}+S_{i}
$$




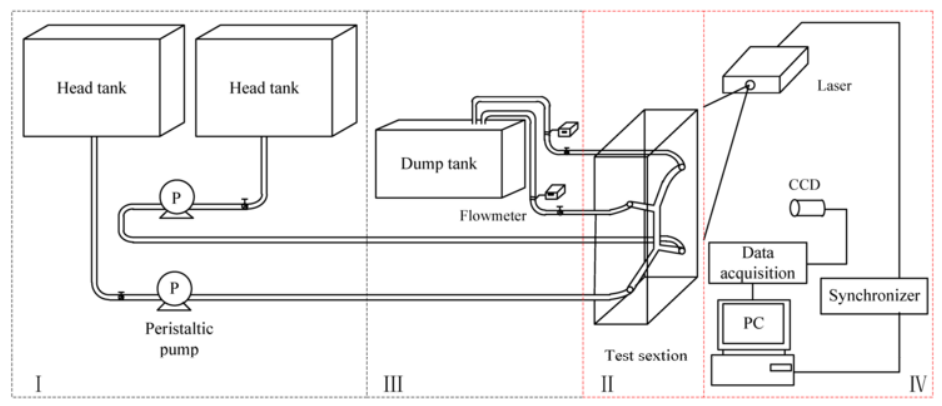

Fig. 3. Schematic of the experimental system.

where $Y_{i}$ and $\vec{J}_{i}$ are the mass fraction and diffusion flux, and $R_{i}$ and $S_{i}$ are the chemical reaction rate and generation rate of the species, respectively. $Y_{i}$ represents the mass fraction of the spleen-derived blood with a SV inlet value of $100 \%$ and a SMV inlet value of $0 \%$. In the present model, $R_{i}$ and $S_{i}$ are neglected because there is no chemical reaction or source term. Since hepatotrophic factors exist only in spleen-derived blood, the percentage of the spleen-derived blood directed into the left and right hepatic lobes can be calculated by spleen-derived blood diffusion in different models, which will allow verification of the hepatotrophic factors hypothesis.

\section{Experiment to verify the numerical algorithm}

We conducted an experiment to validate the numerical algorithm with the results of the idealized model. Figure 3 describes the experimental facility schematically. Two peristaltic pumps with lowpulsation heads (DMD25, Lead Fluid, China) produced a constant flow of working fluid from two tanks. The flow rate ranged from 289.5 to $906.2 \mathrm{~mL} / \mathrm{min}$ with an error of less than $1 \%$. Working fluid was delivered to the transparent idealized PV model made of polymethylmethacrylate (PMMA). We used particle image velocimetry (PIV, TSI, USA) to measure the flow field in the idealized model with different inlet velocities (SMV: 0.02-0.26 m/s; SV: 0.15-0.23 m/s). After flowing out of the test section, the working fluid collected in the dump tank. Since blood easily denatures, we used a $40 \%$ aqueous solution of glycerol as the working fluid, whose physical properties (density and viscosity) and refractive index are similar to those of blood [14].

The curvature of the PV model can cause severe optical distortions during flow imaging. To mitigate these distortions, we immersed the model in a transparent glycerin-filled rectangular test section with flat faces. This method matched the refractive indexes of the solution and the model, effectively reducing optical distortions.

\section{Results and discussions}

\subsection{Experiment validation of the numerical algorithm}

Figures 4 and 5 show streamlines in two different cases to compare the PIV measurements with the numerical simulation calculated using CFD. As displayed in the figures, the numerical simulations largely agree with the experimental results, validating our numerical algorithm. Small fluctuations 


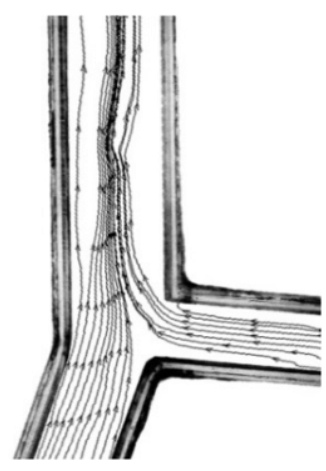

(a) PIV measurement result

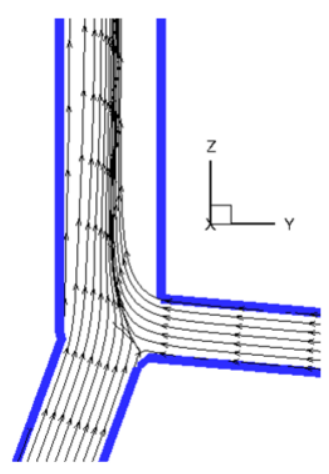

((b) CFD simulation

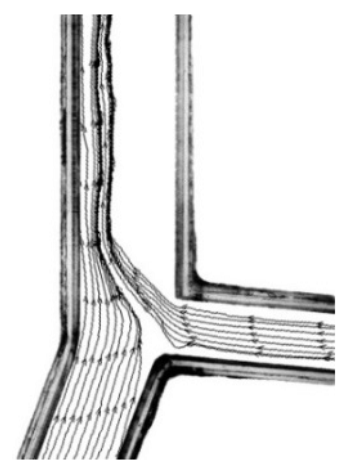

(a) PIV measurement result

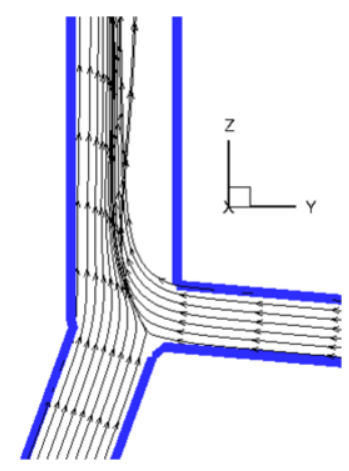

(b) CFD simulation

Fig. 4. Streamline diagrams of case 1 (SMV inlet velocity $0.20 \mathrm{~m} / \mathrm{s}$, SV inlet velocity $0.15 \mathrm{~m} / \mathrm{s}$ ).

Fig. 5. Streamline diagrams of case 2 (SMV inlet velocity $0.22 \mathrm{~m} / \mathrm{s}$, SV inlet velocity $0.23 \mathrm{~m} / \mathrm{s}$ ).

were observed in the PIV results due to the non-ideal flat distribution of inlet flow and measurement error.

\subsection{Idealized models}

\subsubsection{Control group}

Figure 6 describes the influence of SV inlet velocity on the blood velocity distribution in the idealized model of the PV. As shown in the figure, PV velocity increased as SV inlet velocity increased. As SV inlet velocity increased, the backflow region near the SV in Figure 4. (blue area) increased due to more intense mixing of blood. In addition, blood velocity in the RPV significantly increased as SV inlet velocity increased, because the increase in SV inlet velocity led to a corresponding increase in PV velocity with the peak velocity shifting to the right side of the PV (Figure 7). The peak velocity shifted because of the impact of increasing SV inlet velocity on the PV flow.

Figures 8 and 9 illustrate the influence of SMV inlet velocity on the PV velocity distribution. As shown in the figure, PV velocity increased as SMV inlet velocity increased, and the peak velocity shifted to the left side of the PV as SMV inlet velocity increased. The increase in PV velocity occurred due to the increased blood flow in the PV system, and the peak velocity shifted because of the impact of the increasing SMV inlet velocity on the PV flow.

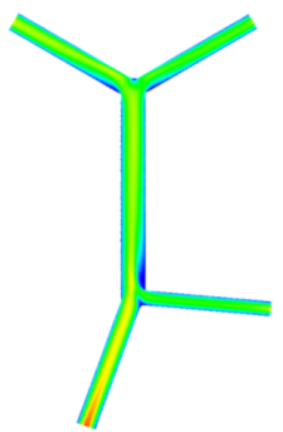

(a) $\mathrm{U}_{\mathrm{SV}}=0.15 \mathrm{~m} / \mathrm{s}$

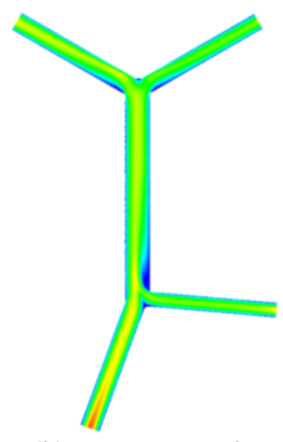

(b) $\mathrm{U}_{\mathrm{SV}}=0.17 \mathrm{~m} / \mathrm{s}$

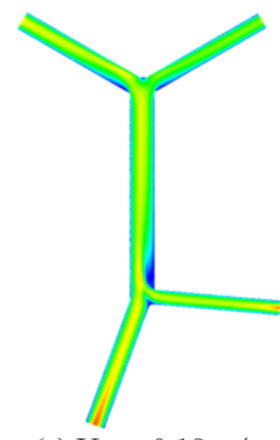

(c) $\mathrm{U}_{\mathrm{SV}}=0.19 \mathrm{~m} / \mathrm{s}$

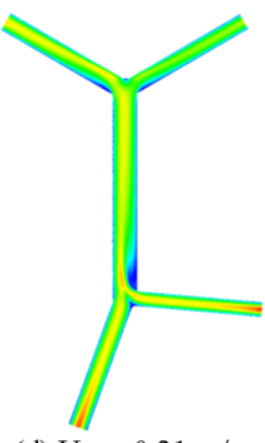

(d) $\mathrm{U}_{\mathrm{SV}}=0.21 \mathrm{~m} / \mathrm{s}$

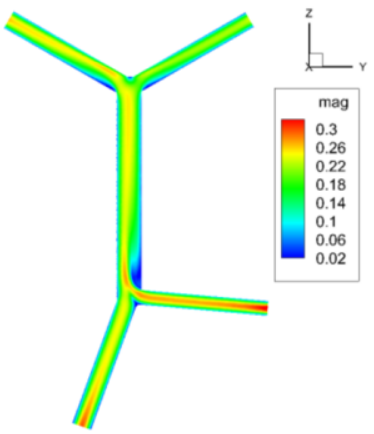

(e) $U_{\mathrm{SV}}=0.23 \mathrm{~m} / \mathrm{s}$

Fig. 6. Velocity distribution with increase of SV flow velocity. 


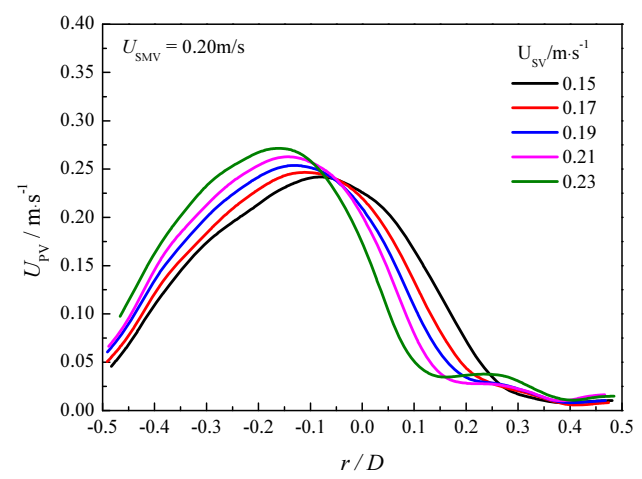

Fig. 7. Velocity distribution at PV inlet with increase of SV flow velocity.

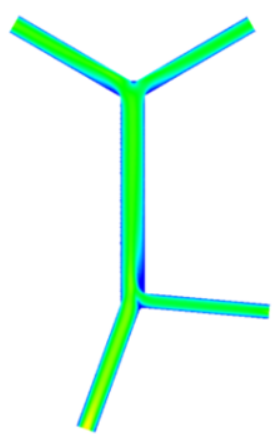

(a) $\mathrm{U}_{\mathrm{SMV}}=0.20 \mathrm{~m} / \mathrm{s}$

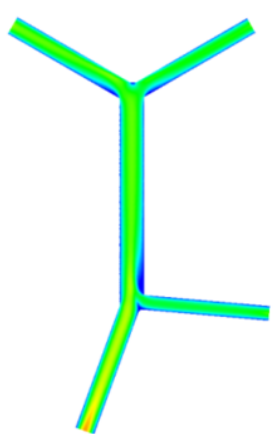

(b) $\mathrm{U}_{\mathrm{SMV}}=0.22 \mathrm{~m} / \mathrm{s}$

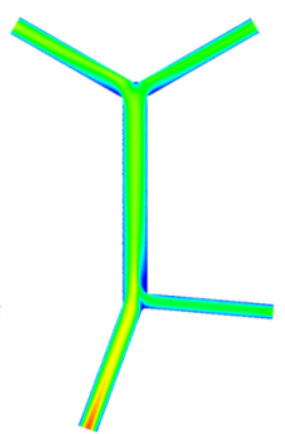

(c) $\mathrm{U}_{\mathrm{SMV}}=0.24 \mathrm{~m} / \mathrm{s}$

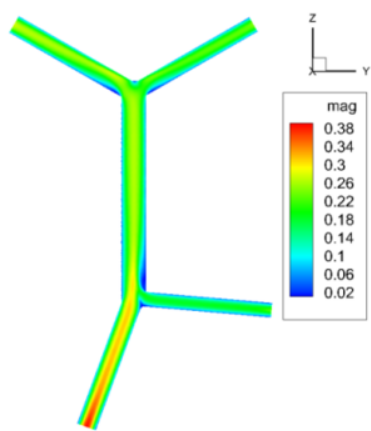

(d) $\mathrm{U}_{\mathrm{SMV}}=0.26 \mathrm{~m} / \mathrm{s}$

Fig. 8. Velocity distribution with increase of SMV flow velocity.

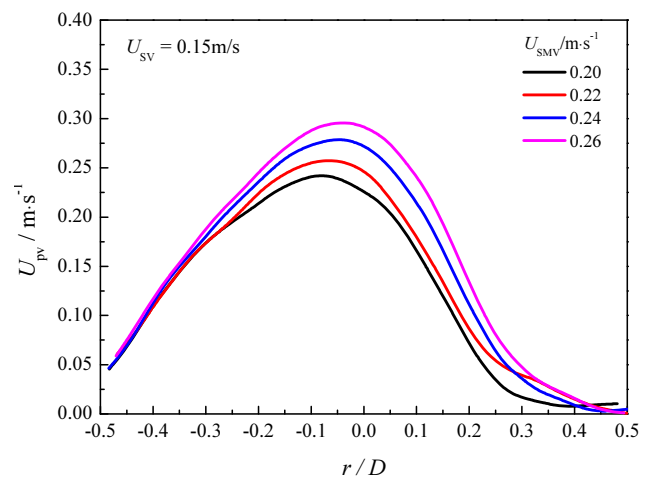

Fig. 9. Velocity distribution at PV inlet with increase of SMV flow velocity.

Using the control group's idealized models, we calculated the diffusion of spleen-derived blood to validate the hepatotrophic factors hypothesis. As shown in Figures 10(a) and 10(b), the numerical results from the idealized models proved that the length and diameter of the PV exerted an important influence on the distribution of spleen-derived blood from the SV. With the increase in PV length, the mass fraction of spleen-derived blood increased in the RPV and decreased in the LPV. Moreover, the mass fraction of blood from the SV decreased in the RPV and increased in the LPV when the diameter of PV increased ( $\mathrm{PV}$ diameter $>13 \mathrm{~mm}$ ). However, the diameter was less closely related to the 
distribution of spleen-derived blood than was the inlet flow rate. As shown in Figures 10(c) and 10(d), neither the angle between the SV and SMV nor the angle between the RPV and LPV had a significant effect on the distribution of spleen-derived blood when they were symmetric distribution.

As illustrated in Figure 11, the control group results showed that the mass fraction of spleen-derived blood in the RPV and LPV increased linearly with an increase in the SV flow rate and decreased linearly with an increase in the SMV flow rate, due to the respective increase and decrease in the flow of spleen-derived blood in the PV system.

\subsubsection{The cirrhosis group}

Compared with the control group, geometric changes in the cirrhosis group are mainly reflected in

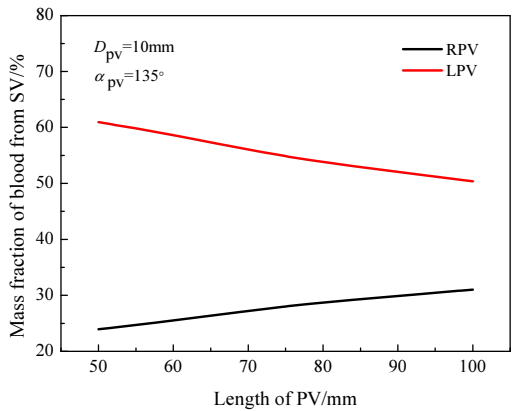

(a) Length of PV

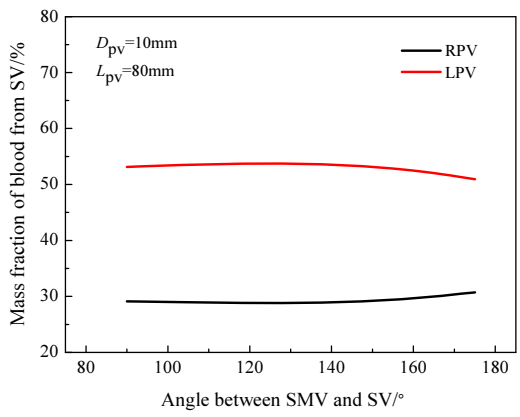

(c) Angle between SMV and SV

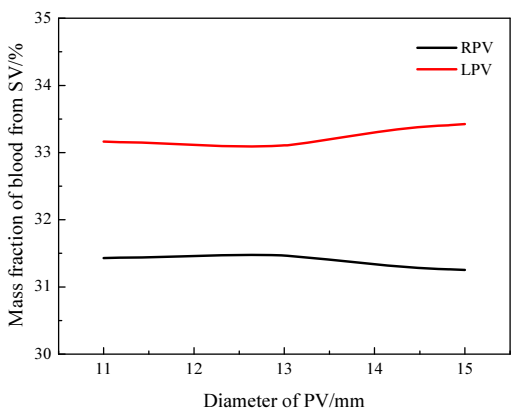

(b) Diameter of PV

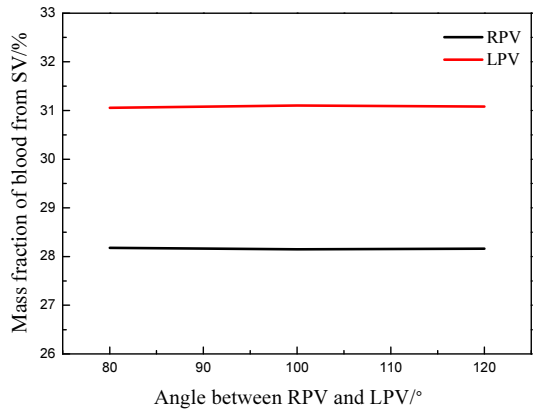

(d) Angle between RPV and LPV

Fig. 10. Distribution of blood from the SV in control group models with different forms.

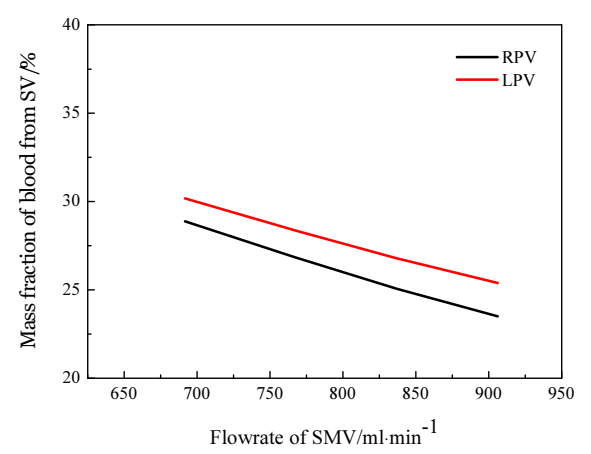

(a) Flow rate of SMV

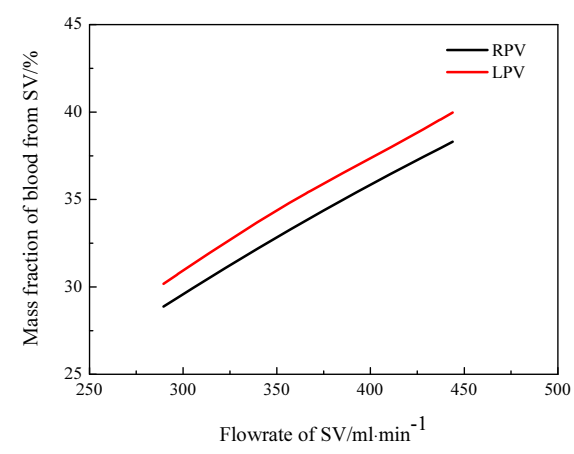

(b) Flow rate of $\mathrm{SV}$

Fig. 11. Distribution of blood from the SV in control group models with different flow rates. 


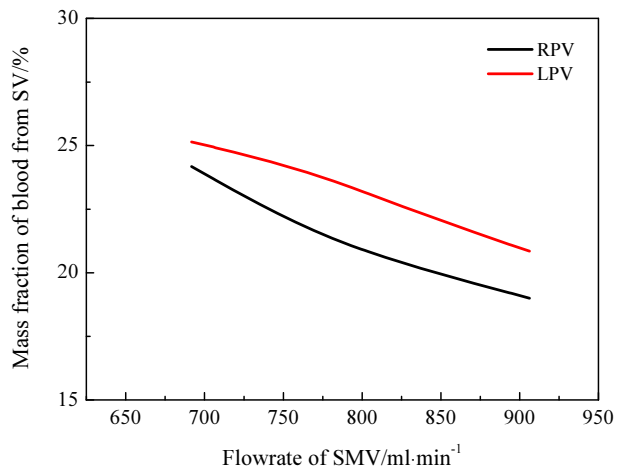

(a) Flow rate of SMV

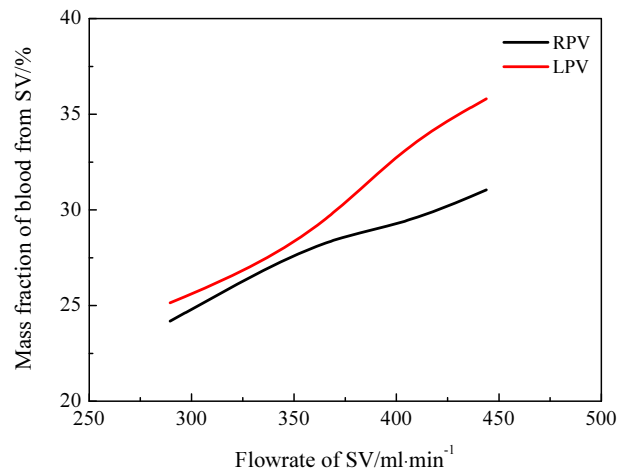

(b) Flow rate of SV

Fig. 12. Distribution of blood from the SV in the cirrhosis group models with different flow rates.

the vasodilation of the PV, SMV and SV due to portal hypertension. The inlet flow setting in the cirrhosis group was consistent with the control group. As shown in Figure 12, results from the cirrhosis patient models showed that the mass fraction of blood from the SV was similar in the RPV and LPV when the inlet flow rate remained low. As the SV flow rate increased, the mass fraction of blood from the SV in the LPV increased more rapidly than in the RPV. When the SMV flow rate increased, the mass fraction of blood from the SV initially fell faster in the RPV than in the LPV. Combining inlet flow rate data from the literature with the above analysis, it can be concluded that PV hypertension leads to an increase in the mass fraction of blood from the SV in both the RPV and LPV, although it increases much faster in the LPV than in the RPV. As illustrated in Figure 12, the mass fraction of spleen-derived blood in liver cirrhosis patients does not change linearly like in the control group shown in Figure 11, due to the vasodilation of the PV, SMV and SV caused by portal hypertension.

\subsection{Patient-specific models}

The CT images showed that vessel geometry varied among the cirrhosis patients as well as the control group. As illustrated in Figure 2, the SV diameter was much larger than the SMV diameter in cirrhosis patients, while opposite was true in the control group. Figure 13 shows differences in blood distribution among control group subjects and the cirrhosis patients. Significant changes in the mass fraction of blood from the SV occur with portal hypertension. Table 2 shows specific results for each subject. The PV structures of control group subjects showed certain differences, but the distributions of spleen-derived blood had a similar right-left branch ratio of 1.2-1.5, with the exception of Control 3. Control 3 differed from other control group subjects due to a very short PV trunk. As shown in Table 2, the two liver cirrhosis patients had larger fractions of spleen-derived blood than control group subjects. The right lobe spleen-derived blood concentration of Patient 1 differed little from the control group, but the left lobe spleen-derived blood concentration increased significantly. The spleen-derived blood concentrations Patient 2's right and left hepatic lobes increased substantially compared with the control group. The right-left branch spleen-derived blood ratio in Patient 1 and Patient 2 was 0.7-0.8, very different from the control group. These results demonstrate that the distribution of spleen-derived blood changes greatly in patients with liver cirrhosis. 


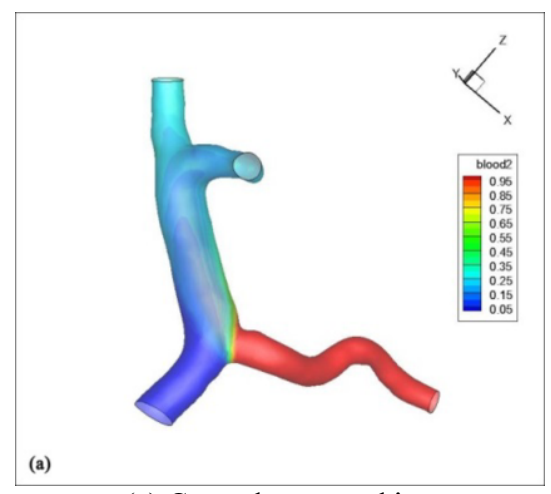

(a) Control group subject

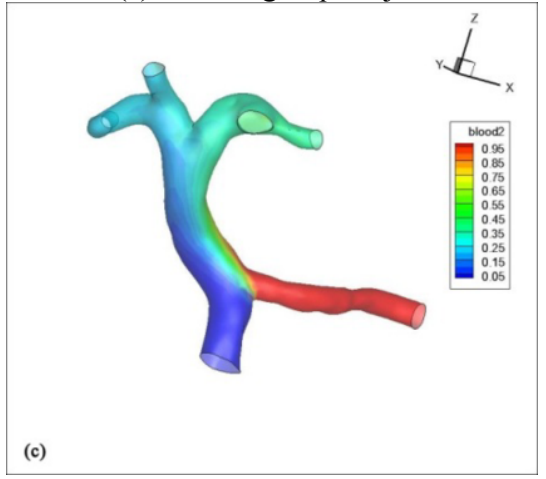

(c) Cirrhosis patient

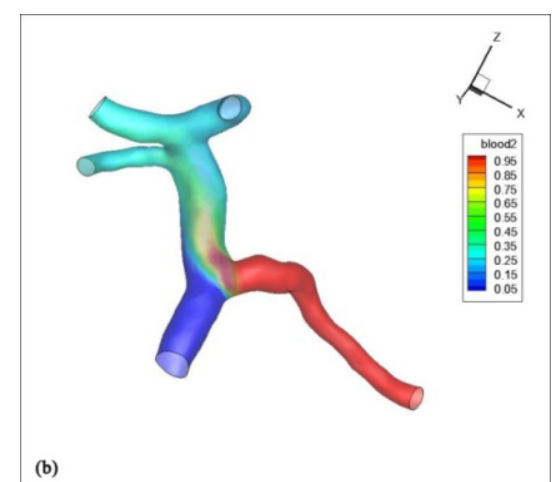

(b) Control group subject 2

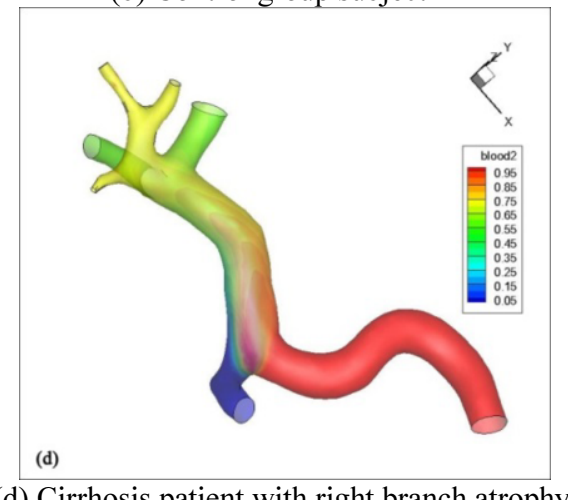

(d) Cirrhosis patient with right branch atrophy

Fig. 13. Contours of mass fraction of blood from the SV.

Table 2

Distribution of blood from the SV in the liver

\begin{tabular}{llll}
\hline Subjects & \multicolumn{3}{l}{ Mass fraction of spleen-derived blood } \\
\cline { 2 - 4 } & RPV & LPV & RPV/LPV \\
\hline Control 1 & $26.72 \%$ & $18.05 \%$ & 1.48 \\
Control 2 & $27.19 \%$ & $22.28 \%$ & 1.22 \\
Control 3 & $25.93 \%$ & $27.09 \%$ & 0.96 \\
Control 4 & $19.93 \%$ & $13.66 \%$ & 1.46 \\
Patient 1 & $25.09 \%$ & $35.01 \%$ & 0.72 \\
Patient 2 & $58.99 \%$ & $72.00 \%$ & 0.82 \\
\hline
\end{tabular}

\section{Conclusion}

This study focused on the simulation of blood flow in PV system models in order to validate the hypothesis that changes in the routing of hepatotrophic factors through the LPV and RPV due to portal hypertension cause the right hepatic lobe atrophy and left hepatic lobe hypertrophy characteristic of advanced cirrhosis. We conducted in vitro experimentation and computational fluid dynamics (CFD) simulation for both an idealized model and specific models based on the CT images acquired from four healthy subjects and two patients with liver cirrhosis. The numerical results agreed well with the experimental measurements, validating the CFD algorithm. Modeling of the control group and the cirrhosis group demonstrated that changes to PV, SMV, and SV diameters, SV inlet flow rates and 
SMV inlet flow rate lead to obvious changes to the mass fraction of spleen-derived blood flow to the right and left hepatic lobes. The flow rate increase characteristic of portal hypertension causes the mass fractions of spleen-derived blood in the RPV and LPV to increase, with a greater increase occurring in the LPV than in the RPV. Simulation of specific subject results confirmed this conclusion. Subject-specific results showed that the mass fraction of spleen-derived blood in the RPV was about 1.2-1.5 times that of the LPV in healthy subjects, with the ratio reduced to $0.7-0.8$ in cirrhosis patients. These results confirm the hypothesis that the distribution of spleen-derived blood in the PV system significantly influences right-lobe atrophy.

\section{Acknowledgments}

This research is supported by the National Science Foundation of China (51176152), Specialized Research Fund for the Doctoral Program of Higher Education (20120201110070) and the Fundamental Research Funds for the Central University.

\section{References}

[1] K.H. Liang and S.B. Li, Portal hypertension, People's Military Medical Press, Beijing, 1999, p. 5.

[2] H. Cichoż-lach, K. Celiński, M. Słomka, et al., Pathophysiology of portal hypertension, Journal of Physiology and Pharmacology 59 (2008), 231-238.

[3] H. Ho, A. Bartlett and P. Hunter, Hemodynamic simulation for an anatomically realistic portal system, Medical Image Computing and Computer-Assisted Intervention, 2011, Springer Berlin Heidelberg, pp. 347-354.

[4] N. Toubia and A.J. Sanyal, Portal hypertension and variceal hemorrhage, Medical Clinics of North America 92 (2008), 551-574.

[5] T.D. Boyer, T.L. Wright and M.P. Manns, Zakim and Boyer's Hepatology, A Textbook of Liver Disease, Elsevier Health Sciences, Saunders, Philadelphia, 2011.

[6] X.Y. Yin, M.D. Lu, J.F. Huang, et al., Color Doppler velocity profile assessment of portal hemodynamics in cirrhotic patients with portal hypertension: Correlation with esophageal variceal bleeding, Journal of Clinical Ultrasound 29 (2001), 7-13.

[7] Y. Yamagishi, H. Saito, S. Tada, et al., Value of computed tomography-derived estimated liver volume/standard liver volume ratio for predicting the prognosis of adult fulminant hepatic failure in Japan, Journal of Gastroenterology and Hepatology 20 (2005), 1843-1849.

[8] M. Stockman, J. Lock, R. Rohl, et al., Liver function and volume regeneration after hepatectomy, Journal of Hepatology 1 (2009), S185.

[9] M.Q. Xu, L.N. Yan, B. Li, et al., Initial clinical effect of intraportal insulin administration on liver graft regeneration in adult patients underwent living donor right lobe liver transplantation, Transplantation Proceedings 41 (2009), 16981702.

[10] T.L. Hwang, M.F. Chen and T.J. Chen, Augmentation of liver regeneration with glucagon after partial hepatectomy in rats, Journal of the Formosan Medical Association 92 (1993), 725-728.

[11] C.C. Botar, T. Vasile and S. Sfrangeu, et al., CFD simulation of the portal vein blood flow, International Conference on Advancements of Medicine and Health Care through Technology, Springer Berlin Heidelberg, 2009, pp. 359-362.

[12] S. Petkova, A. Hossain, J. Naser, et al., CFD modelling of blood flow in portal vein hypertension with and without thrombosis, Third International Conference on CFD in the Minerals and Process Industries, Melbourne, Australia, 2003, pp. 527-530.

[13] S.M. George, Hemodynamic investigation of the liver using magnetic resonance imaging and computational fluid dynamics, Ph.D. Dissertation, Georgia Institute of Technology, 2008.

[14] M. Jan, V. Katerina and A. Josef, Using the PIV method for blood flow investigation, Congress of the Czech Society and Biomechanics, Czech, 2004. 